\title{
Assessment of the hyperbaric oxygen therapy effects in distal femoral necrosis using hyperspectral imaging
}

\author{
CALIN Mihaela Antonina' ${ }^{1}$, MACOVEI Adrian², MANEA Dragos ${ }^{1}$
}

Editor: Constantin MUNTEANU, E-mail: office@bioclima.ro

Corresponding author: CALIN Mihaela Antonina, E-mail: mantonina calin@yahoo.com; micalin@inoe.inoe.ro

1. "National Institute of Research and Development for Optoelectronics INOE 2000", Magurele, Romania 2. “„Gen. Dr. Aviator Victor Anastasiu” National Institute of Aeronautical and Space Medicine”, Bucharest, Romania

\begin{abstract}
Introduction. Hyperbaric oxygen therapy is a treatment option for an increasing number of conditions. The aim of this study was to assess its therapeutic effects in femoral condylar necrosis as well as in normal tissue using hyperspectral imaging. Material and method. A 47-year-old female patient diagnosed with avascular necrosis of the medial femoral condyle was included in this study. The patient received hyperbaric oxygen therapy for two consecutive days (the first day: three stages of $30 \mathrm{~min}$ each, separated by two breaks of 5 min at $\mathrm{pO}_{2}=2$ ATA; the second day: three stages of $30 \mathrm{~min}$ each, separated by two breaks of $5 \mathrm{~min}$ at $\mathrm{pO}_{2}=2.4$ ATA). The effects of hyperbaric oxygen therapy on the affected area (patient's knee) and normal (patient's hand) tissues oxygenation were assessed before and after each treatment session using hyperspectral imaging. Results and discussions. The results showed that hyperspectral imaging is quite good for monitoring the hyperbaric oxygen therapy efficacy in affected areas, even with deep tissue problems. Conclusions. In conclusion, the hyperspectral imaging-based approach for monitoring hyperbaric oxygen therapy could support physicians in extracting prognostic information and making the right treatment decisions.
\end{abstract}

Keywords: bone necrosis, image analysis, maps, oxyhemoglobin, deoxyhemoglobin, oxygen saturation,

\section{INTRODUCTION}

Hyperbaric oxygen therapy (HBOT) is a therapeutic modality that consists of the administration of high concentrations of pure oxygen, at pressures exceeding atmospheric pressure $(101.325 \mathrm{kPa})$, in patients placed inside a hyperbaric chamber, for therapeutic purposes. The action mechanism of this therapeutic modality is a complex one and it has been proven that it can lead to a series of biochemical, cellular and physiological effects (1) that could be exploited for the treatment of several different diseases. Nowadays, the HBOT therapy is recommended as the main or complementary therapy for: decompression sickness and $\mathrm{CO}$ poisoning (2), gas embolism (3), necrotizing soft tissue infections (4), diabetic foot lesions (5), femoral head necrosis (6), radiation injury (7), burns (8), and acute anemia (9). The effects of hyperbaric oxygen therapy are usually assessed using methods such as: pulse oximetry $(10,11)$, transcutaneous oximetry $(12,13)$, thermal imaging and planimetry (14) or magnetic resonance imaging for lesion progressing (15). However, for a more accurate quantitative assessment of the HBOT therapy effects and a better understanding of its action mechanism at the tissular level, new non-invasive methods with real-time applications are still required.

This paper proposes a new approach for assessing the effects of HBOT therapy on tissue oxygenation based on hyperspectral imaging (HSI). The hyperspectral imaging technique (also known as imaging spectroscopy) introduced by Goetz (16) in the late 1980s for Earth observations has more recently aroused interest in the medical field for: cancer detection, diabetic foot ulcer prognosis, peripheral vascular disease assessment, burn characterization, etc. $(17,18)$. This is because the HSI technique, by combining the facilities offered by two optical methods (digital imaging and spectroscopy), has the ability to simultaneously provide spatial and spectral information about an investigated area, which can be particularly useful to support clinicians in diagnosing various pathologies or monitoring the treatment efficiency. 
The aim of this paper was to explore the possibility of using spectral-spatial information provided by hyperspectral imaging, via a chemometric regression approach, to generate distribution maps of tissue constituents (oxyhemoglobin and deoxyhemoglobin) in the investigated areas (pathologic-knee and normalhand). These maps can be considered as new tools for non-invasive assessment and easier interpretation of the effects of HBOT therapy.

The main objectives of this study were: (1) setting the conditions for hyperspectral images acquisition; (2) establishing the appropriate method for preprocessing hyperspectral images; (3) analysis of hyperspectral data and generation of distribution maps of oxyhemoglobin and deoxyhemoglobin concentrations in affected and adjacent areas exposed to HBOT therapy; and (4) interpretation of results with the extraction of prognostic information and orientations towards therapeutic options.

\section{Materials and Methods}

\subsection{Patient}

A 47-year-old female patient (height $=168 \mathrm{~cm}$, weight $=$ $95 \mathrm{~kg}$ ) diagnosed with avascular necrosis of the medial femoral condyle was selected for this preliminary study. An informed consent was obtained from the patient prior to participation in the study. The procedures performed in this study involving human participants were in accordance with the ethical standards of the "Gen. Dr. Aviator Victor Anastasiu" National Institute of Aeronautical and Space Medicine Research and Ethics Committee and with the 1964 Helsinki declaration and its later amendments or comparable ethical standards. On clinical examination, the patient presented pain, function limitation and swelling of the knee. Diagnosis was confirmed by magnetic resonance imaging investigation. HBOT therapy was recommended as a possible treatment by an orthopedic surgeon.

\subsection{Hyperbaric oxygen therapy (HBOT)}

Hyperbaric oxygen therapy was performed using a BARAMED monoplace hyperbaric chamber (ETC, Southampton, USA). The patient received HBOT once a day for several days, the first two days being selected for this study. On the first day, the patient was exposed to $\mathrm{HBOT}$ inside the hyperbaric chamber at a $\mathrm{O}_{2}$ pressure of 2 absolute atmospheres (ATA) for $90 \mathrm{~min}$, in three stages of $30 \mathrm{~min}$ each, separated by two breaks of $5 \mathrm{~min}$ breathing ambient air. Oxygen was administered by an on-demand regulator on a standard care mask. The next day, the procedure was repeated, but at an $\mathrm{O}_{2}$ pressure increased to 2.4 ATA.

The immediate effects of HBOT therapy on the normal and pathological tissues oxygenation were evaluated before and after each treatment session using hyperspectral imaging.

\subsection{Hyperspectral images acquisition}

Hyperspectral images of the patient's hand and knee (normal and pathological tissues, respectively) were acquired using a line-scanning hyperspectral imaging system. This system consists of: (1) a hyperspectral sensor (ImSpector V8E, Specim, Oulu, Finland) that allows the simultaneous acquisition of 205 spectral bands in the spectral range $(400-800) \mathrm{nm}$, at a spectral resolution of $1.95 \mathrm{~nm}$; (2) an illumination unit consisting of two 300W halogen lamps (OSRAM, Munich, Germany) equipped with diffusion filters for uniform illumination of the area of interest; (3) a galvanometric scan mirror (GVS211, Thorlabs, New Jersey, USA) for scanning of the region of interest; (4) a computer with specialized software installed for the acquisition, processing and analysis of hyperspectral data, such as SpectralDAQ software (Specim, Oulu, Finland) and ENVI v.5.1 software (Exelis Visual Information Solutions, Boulder, Colorado, USA), and (5) a tripod (Manfrotto, Cassola, Italy) for mounting the hyperspectral sensor in a suitable position to acquire the image of a region of interest (Fig 1.)

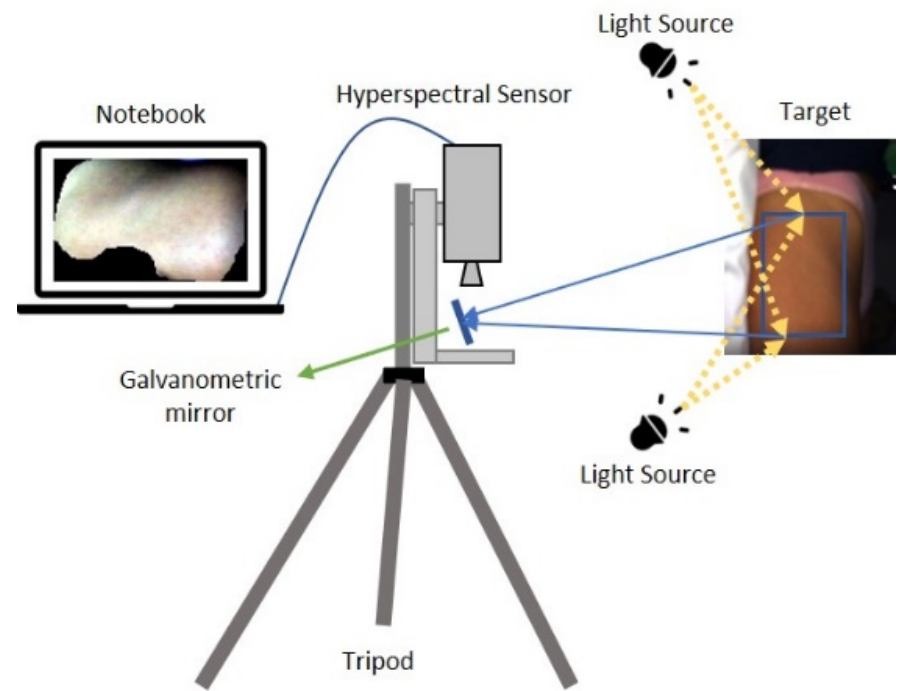

Fig. 1. The experimental setup used for the acquisition of normal and pathological tissues hyperspectral images Hyperspectral images acquired with this hyperspectral system often contain artifacts and aberrations associated with the type of line-by-line scanning that affects image quality. Therefore, prior to any further analysis of hyperspectral images, a processing step of the original data is required.

\subsection{Hyperspectral images processing}

The processing of the original hyperspectral images was performed in two steps (Fig. 2). The first step was aimed at minimizing the signal variations of the hyperspectral system introduced by the spatial non-uniformity of the artificial light intensity in the scene to be investigated and by the dark current of the monochrome camera. 
All acquired hyperspectral images were calibrated with white and dark reference images, using Equation (1) :

$$
I_{\text {calibrated }}=\frac{I_{\text {original }}-I_{\text {dark }}}{I_{\text {white }}-I_{\text {dark }}}
$$

where: $\mathrm{I}_{\text {calibrated }}$ is the calibrated hyperspectral image, Ioriginal is the original hyperspectral image of the investigated areas, $I_{\text {dark }}$ is the dark reference image acquired by completely covering the hyperspectral sensor lens with its black cap and turning off the illumination unit, and $\mathrm{I}_{\text {white }}$ is the white reference image acquired using a polytetrafluoroethylene (PTFE) reference tile (model WS-2, Avantes, Apeldoorn, Netherlands), located in the area to be investigated (Fig. 2.a).

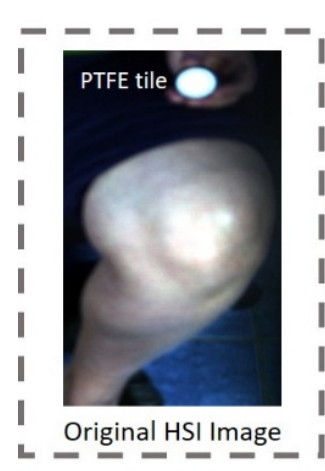

a)

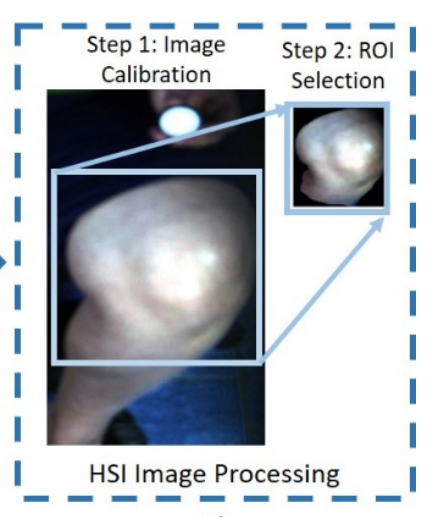

b)
Fig. 2. Hyperspectral image processing flowchart of the patient left leg. a) original image of the patient's leg; b) image processing (image calibration and ROI selection relative only to the patient's knee)

The high dimensionality of hyperspectral data was reduced in the second step by selecting a region of interest (ROI) relative only to the area to be investigated (hand/knee) and eliminating other elements present in the scene, not relevant to the area to be investigated which would complicate the subsequent analysis of the data (Fig. 2.b). Image processing was repeated at the beginning of each experimental measurement, with the white reference standard placed in the same scene as the investigated area. Hyperspectral images acquired before and after exposure of patients to HBOT therapy, after appropriate processing, were used as a basis for subsequent chemometric analysis of chromophores identified in the investigated tissues.

\subsection{Hyperspectral data analysis}

The spatial and spectral information contained in the processed hyperspectral images was exploited to generate distribution maps of oxyhemoglobin $\left(\mathrm{HbO}_{2}\right)$ and deoxyhemoglobin $(\mathrm{Hb})$ concentrations in biological tissues using an approach based on Beer-Lambert law
(19) and Levenberg-Marquardt method (20). The approach assumes, as a first step, the conversion of hyperspectral images (reflectance images, $R(\lambda)$ ) into absorbance images $A_{\text {calc }}(\lambda)$ using Equation (2):

$$
A_{\text {calc }}(\lambda)=-\log _{10} R(\lambda)
$$

In the next step, the absorbance $A_{\text {est }}(\lambda)$ in each pixel of the image is estimated according to the Beer-Lambert law, by Equation (3):

$$
\mathrm{A}_{\text {est }}(\lambda)=\boldsymbol{\varepsilon}_{\text {oxy }}(\lambda) \mathrm{C}_{\text {oxy }}+\boldsymbol{\varepsilon}_{\text {Dexy }}(\lambda) \mathrm{C}_{\text {Dexxy }}+\mathrm{T}
$$

where: $\mathrm{C}_{\mathrm{Oxy}}$ and $\mathrm{C}_{\text {Deoxy }}$ represent the surface molar concentrations of $\mathrm{HbO}_{2}$ and $\mathrm{Hb}[(\mathrm{mol} / \mathrm{L}) \cdot \mathrm{cm}], \varepsilon_{\text {Oxy }}$ and $\varepsilon_{\text {Deoxy }}$ are the extinction coefficients $\left(\mathrm{cm}^{-1} /(\mathrm{mol} / \mathrm{liter})\right)$ of $\mathrm{HbO}_{2}$ and $\mathrm{Hb}$, and $\mathrm{T}$ is a term that takes into account the effect of light scattering. Finally, the terms $\mathrm{C}_{\text {oxy }}$ are $\mathrm{C}_{\text {Deoxy }}$ are determined by minimizing the sum of square difference between the calculated absorbance $\mathrm{A}_{\text {calc }}$ and the estimated absorbance $\mathrm{A}_{\text {est }}$ using the nonlinear leastsquare method (Eq. 4).

$$
\sum_{\mathrm{k}}\left[\mathrm{A}_{\text {calc }}(\lambda)-\mathrm{A}_{\text {est }}(\lambda)\right]^{2} \rightarrow \min
$$

The minimization of the function described by Eq. 4 is performed using the Levenberg-Marquardt method.

The results of this approach are distribution maps of oxyhemoglobin and deoxyhemoglobin concentrations generated both as images (.tif files) and as numerical data (ASCII text files). In addition, the information contained in these maps can also be exploited to evaluate oxygen saturation $\left(\mathrm{StO}_{2}\right)$ in each pixel of the analyzed region of interest. This parameter is defined by Equation (5):

$$
\mathrm{StO}_{2}=\frac{\mathrm{C}_{\text {oxy }}}{\mathrm{C}_{\text {oxy }}+\mathrm{C}_{\text {Dexy }}}
$$

These results, in the form of distribution maps of $\mathrm{HbO}_{2}$, $\mathrm{Hb}$ concentrations and $\mathrm{StO}_{2}$, allow the analysis of the changes induced by HBOT therapy in the oxygenation level of the investigated pathological area, in a simple and easy to interpret graphical manner.

\section{Results}

Figure 3 shows the distribution maps of oxyhemoglobin and deoxyhemoglobin concentrations, as well as oxygen saturation across the region of interest related to the patient's pathological area (knee) determined from hyperspectral data obtained at different time moments during HBOT treatment (before and after the first and the 
second treatment day). The distribution maps of the coefficient of determination $\left(\mathrm{R}^{2}\right)$ calculated over the same region of interest indicating the accuracy degree of the regression equation adjustment to the experimental data are also shown (Fig. 3.d,h,l,p).

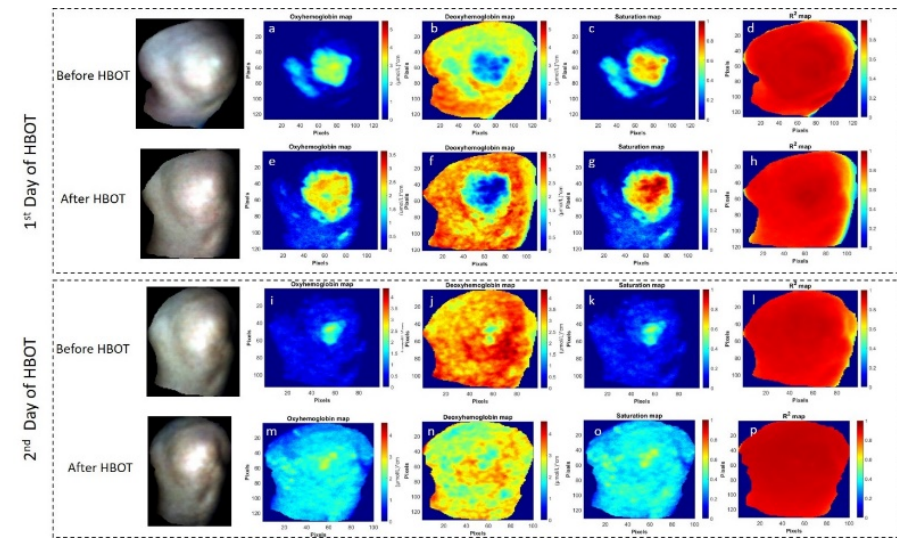

Fig. 3. Distribution maps of oxyhemoglobin and deoxyhemoglobin concentrations, oxygen saturation and

$\mathrm{R}^{2}$ values over the patient's pathological area (knee) calculated from hyperspectral data obtained at different time moments during HBOT treatment (before and after the first and the second treatment day). a), e), 1) and m)

distribution maps of oxyhemoglobin concentrations before and after each HBOT treatment session; b), f), j), and $\mathrm{n}$ ) distribution map of deoxyhemoglobin concentrations before and after each HBOT treatment session; c), g), k), and o) oxygen saturation at different time moments during HBOT treatment; d), h), l), and p) $\mathrm{R}^{2}$ values calculated at different time moments during HBOT treatment

It is noticeable from Figure 3 that the $\mathrm{HbO}_{2}$ concentration increases after the first session of HBOT therapy, especially in the central area of the knee, thus clearly highlighting the affected area, but decreases significantly in the next $24 \mathrm{~h}$. After the second HBOT therapy session, the significant increase in $\mathrm{HbO}_{2}$ concentration observed throughout the patient's knee area can be explained by an improvement in blood circulation in the knee region, probably due to the initiation of the neovascularization process by the mechanism of intermittent hypoxia. The deoxyhemoglobin concentration increases after the first session of HBOT therapy as well as within the $24 \mathrm{~h}$ interval between treatment days, an effect just attributable to the intermittent hypoxia mentioned above, with the establishment of the neovascularization process. The second HBOT session led to only a small decrease in deoxyhemoglobin concentration, due to persistent neovascularization that did not change during exposure. The $\mathrm{R}^{2}$ values very close to 1 found in all the analyzed cases indicate a high degree of correlation between the calculated and experimental data.
In the case of normal tissues, the effect of HBOT therapy is much more pronounced as seen in Figure 4. Possible explanations could be related to the different vascular structure of the examined areas. Taking into account the patient's pathology, although the lesion is deep (bone) it is likely that some pathological changes in the superficial circulation already exist which could justify the oxygenation state of the patient's hand).
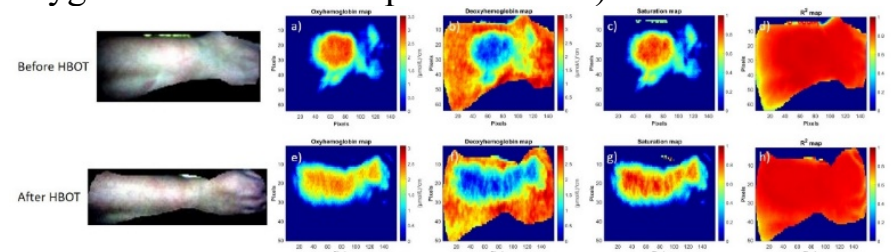

Figure 4. Distribution maps of $\mathrm{HbO}_{2}$ and $\mathrm{Hb}$ concentrations, $\mathrm{StO}_{2}$ and $\mathrm{R}^{2}$ values in normal tissue (patient's hand) before and after a HBOT treatment session. a), e) distribution maps of oxyhemoglobin concentrations before and after HBOT treatment; b), f) distribution map of deoxyhemoglobin concentrations

before and after HBOT treatment; c), g) oxygen saturation before and after HBOT treatment; d), h) $\mathrm{R}^{2}$ values calculated before and after HBOT treatment The changes in the oxygen content of the normal and pathological areas induced by HBOT session, expressed in terms of mean values of oxyhemoglobin and deoxyhemoglobin concentrations and oxygen saturation, are shown in Fig. 5.

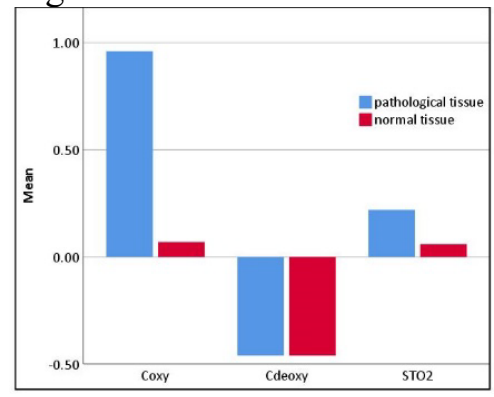

Fig. 5. Difference in the mean values of $\mathrm{HbO}_{2}$ and $\mathrm{Hb}$ concentrations, and $\mathrm{StO}_{2}$ of normal and pathological tissues after HBOT therapy

It should be noted that although in Figs. 3 and 4 there are increases/decreases in chromophores concentrations in certain areas of the knee/hand, considerable differences are recorded only for the mean concentrations of $\mathrm{HBO}_{2}$ and $\mathrm{StO}_{2}$. The variation of the mean concentration of deoxyhemoglobin remains approximately constant $(0.46)$. This could be due to the fragile development of redistributed blood flow in the first HBOT sessions. The effect is expected to be permanent in the affected areas, but only after a significant number of HBOT sessions (usually over 5), which we could not monitor for this study, even if the patient received a full course. Shortterm monitoring excludes chronic circulatory and metabolic changes attributable to hyperbaric oxygen therapy. 
Therefore, $\mathrm{HbO}_{2}$ and $\mathrm{StO}_{2}$ distribution maps generated from hyperspectral images can be considered as useful tools for non-invasive monitoring of the local level of oxygenation of normal and pathological tissues that can be correlated with the evolution of pathology treated by HBOT therapy, thus helping physicians to take correct decisions about the treatment efficiency and staging.

\section{Discussion}

Herein, a new approach for assessing the effects of HBOT therapy on tissue oxygenation based on hyperspectral imaging that has the ability to provide spatial-spectral information that can be used to generate distribution maps of tissue constituents (oxyhemoglobin and deoxyhemoglobin), via a chemometric regression method, was presented. This approach consists mainly of two steps: (1) processing of hyperspectral data (image calibration, ROI selection) and (2) analysis of hyperspectral data using a Beer-Lambert law-based approach and the Levenberg-Marquardt method to generate distribution maps of oxyhemoglobin and deoxyhemoglobin concentrations in biological tissues to facilitate the highlighting of local oxygenation levels of normal and pathological areas, and therefore the local effects of HBOT therapy. Such distribution maps of oxyhemoglobin and deoxyhemoglobin concentrations have seldom been reported so far in clinical practice where pulse oximetry is most widely used method for measuring oxygen saturation levels, but unfortunately of limited use in HBOT. Therefore, the proposed approach could be considered a particularly useful tool for evaluating the effects of HBOT therapy, providing valuable information not only about changes in oxygen saturation levels (that can also be measured by the pulse oximetry method), but also about changes in the oxyhemoglobin and deoxyhemoglobin concentrations in the pathological tissues. Such information gives an opportunity to enrich knowledge about the HBOT therapy mechanism, which is not yet fully understood (21), and at the same time to establish a proper therapeutic schedule for specific pathologies.

In this study, the ability of hyperspectral imaging to assesses the hyperbaric oxygen therapy effects affected areas was assessed as response to the requirement for non-invasive and fast methods to assess the immediate and local effects of HBOT therapy.

The results showed that, through an appropriate analysis of hyperspectral images of the patient's knee and hand, the immediate effects of HBOT therapy could be seen in both pathological and normal areas. Increases in mean oxyhemoglobin concentration and oxygen saturation levels were recorded in all investigated areas, more significant in affected areas than in normal tissues. On the other hand, local deoxyhemoglobin levels decreased after tissues exposure to the same HBOT therapy protocol, but without significant difference between normal and necrotic areas, explained by local vasoconstriction.

Promising results in the use of HBOT therapy for femoral head necrosis have also been reported in several other studies that have highlighted the HBOT therapy effects based on clinical outcome (range of motion, stabilometry and pain $(6)$ or by magnetic resonance imaging $(15,22)$. However, to our knowledge, the use of hyperspectral imaging to assess the effects of HBOT therapy on femoral condylar necrosis is presented for the first time in this study. It has the advantage of providing important information about the local effectiveness of HBOT, and can be considered as a useful monitoring option that can provide evidence of the HBOT effect.

Although this study has shown the role of hyperspectral imaging in assessing the effects of HBOT therapy, for a better understanding of how HBOT therapy may be beneficial in the treatment of bone disease, further studies should be conducted on: (1) validation of the HSI method by comparison with other methods (e.g. transcutaneous oximetry, thermography, planimetry, etc.), but also by using larger groups of patients to confirm its outputs; (2) improving the performance of the algorithm for calculating chromophore concentrations, so as to allow the generation of total hemoglobin maps useful in highlighting the neovascularization process induced by HBOT therapy; and (3) identifying modalities to reduce data analysis time for real-time measurements. All these issues will be addressed in further studies.

\section{Conclusions}

In conclusion, the effects of HBOT therapy on affected and normal areas could be clearly demonstrated by the oxyhemoglobin, deoxyhemoglobin and oxygen saturation maps generated by hyperspectral imaging. The HSI-based approach proposed in this study for assessing the HBOT therapy effects has been shown to be sufficiently accurate both as critical areas identification and as predictor of therapeutic effect and it can be an option for HBOT physicians in making the right treatment decision.

\section{References}

1. Cervaens M, Marques F, Camacho Ó, Barata P. HYPERBARIC OXYGEN THERAPY IN MUSCLE INJURIES. 2010;7:294-304.

2. Shank ES, Muth CM. Decompression illness, latrogenic gas embolism, and carbon monoxide poisoning: The role of hyperbaric oxygen therapy [Internet]. Vol. 38, International Anesthesiology Clinics. Lippincott Williams and Wilkins; 2000 [cited 2021 Apr 6]. p. 111-38. Available from: https://pubmed.ncbi.nlm.nih.gov/10723672/

3. Moon RE. Hyperbaric treatment of air or gas embolism: current recommendations. Undersea Hyperb Med. 2019 Sep $1 ; 46(5): 673-83$ 
4. Steiner T, Seiffart A, Schumann J, Bucher M. Hyperbaric oxygen therapy in necrotizing soft tissue infections: A retrospective study. In: Advances in Experimental Medicine and Biology [Internet]. Springer New York LLC; 2018 [cited 2021 Apr 6]. p. 263-7. Available from: https://pubmed.ncbi.nlm.nih.gov/30178356/

5. Stoekenbroek RM, Santema TB, Legemate DA, Ubbink DT, Van Den Brink A, Koelemay MJW. Hyperbaric oxygen for the treatment of diabetic foot ulcers: A systematic review. Vol. 47, European Journal of Vascular and Endovascular Surgery. W.B. Saunders Ltd; 2014. p. 647-55.

6. Camporesi EM, Vezzani G, Bosco G, Mangar D, Bernasek TL. Hyperbaric oxygen therapy in femoral head necrosis. J Arthroplasty. 2010 Sep 1;25(SUPPL. 6):118-23.

7. Feldmeier JJ. Hyperbaric oxygen for radiation injury: Is it indicated? [Internet]. Vol. 18, Current Oncology. Multimed Inc.; 2011 [cited 2021 Apr 6]. p. 211-2. Available from: /pmc/articles/PMC3185897/

8. Villanueva E, Mh B, Wasiak J, Jp L. Hyperbaric oxygen therapy for thermal burns (Review). [cited 2021 Apr 6]; Available from: http://www.thecochranelibrary.com

9. Graffeo C, Dishong W. Severe blood loss anemia in a Jehovah's Witness treated with adjunctive hyperbaric oxygen therapy. Am J Emerg Med [Internet]. 2013 Apr 1 [cited 2021 Apr 6];31(4):756.e3-756.e4. Available from: http://dx.doi.org/10.1016/j.ajem.2012.11.013

10. KE G, JR H, GA S, KJ H, NS P. Assessment of oxygen saturation levels during a mild hyperbaric chamber treatment. J Clin Investig Stud. 2018;1(2).

11. Martinelli B, Noronha JM, Sette MFM, dos Santos IP, Barrile SR, Simão JC. Cardiorespiratory alterations in patients undergoing hyperbaric oxygen therapy. Rev da Esc Enferm [Internet]. 2019 [cited 2021 Apr 8];53:1-8. Available https://pubmed.ncbi.nlm.nih.gov/31508730/

12. Batenburg MCT, van den Bongard HJGD, Kleynen CE, Maarse W, Witkamp A, Ernst M, et al. Assessing the effect of hyperbaric oxygen therapy in breast cancer patients with late radiation toxicity (HONEY trial): a trial protocol using a trial within a cohort design. Trials [Internet]. 2020 Dec 1 [cited 2021 Apr 8];21(1):980. Available from: https://trialsjournal.biomedcentral.com/articles/10.1186/s13 063-020-04869-z

13. Kaur S, Pawar M, Banerjee N, Garg R. Evaluation of the efficacy of hyperbaric oxygen therapy in the management of chronic nonhealing ulcer and role of periwound transcutaneous oximetry as a predictor of wound healing response: A randomized prospective controlled trial. J Anaesthesiol Clin Pharmacol [Internet]. 2012 Jan [cited 2021 Apr 8];28(1):70-5. Available from: /pmc/articles/PMC3275977/

14. Englisz-Jurgielewicz B, Cholewka A, Firganek E, Knefel G, Kawecki M, Glik J, et al. Evaluation of hyperbaric oxygen therapy effects in hard-to-heal wounds using thermal imaging and planimetry. J Therm Anal Calorim [Internet]. 2020 Aug 1 [cited 2021 Apr
8];141(4):1465-75

Available

from:

https://doi.org/10.1007/s10973-019-09129-0

15. Reis ND, Schwartz O, Militianu D, Ramon Y, Levin D, Norman D, et al. Hyperbaric oxygen therapy as a treatment for stage-I avascular necrosis of the femoral head. J Bone Jt Surg - Ser B [Internet]. 2003 Apr [cited 2021 Apr 8];85(3):371-5. Available from: https://pubmed.ncbi.nlm.nih.gov/12729112/

16. Goetz AFH. Three decades of hyperspectral remote sensing of the Earth: A personal view. Remote Sens Environ. 2009 Sep 1;113(SUPPL. 1):S5-16.

17. Lu G, Fei B. Medical hyperspectral imaging: a review. J Biomed Opt [Internet]. 2014 Jan 20 [cited 2021 Apr 8];19(1):010901. Available from: https://pubmed.ncbi.nlm.nih.gov/24441941/

18. Calin MA, Parasca SV, Savastru D, Manea D. Hyperspectral imaging in the medical field: Present and future. Appl Spectrosc Rev [Internet]. 2014 Aug 18 [cited 2021 Apr 8];49(6):435-47. Available from: https:/www.tandfonline.com/doi/abs/10.1080/05704928.20 13.838678

19. Maikala R V. Modified Beer's Law - historical perspectives and relevance in near-infrared monitoring of optical properties of human tissue. Int J Ind Ergon. 2010 Mar;40(2):125-34.

20. Croeze A, Pittman L, Reynolds W. SOLVING NONLINEAR LEAST-SQUARES PROBLEMS WITH THE GAUSS-NEWTON AND LEVENBERGMARQUARDT METHODS.

21. Sunkari VG, Lind F, Botusan IR, Kashif A, Liu ZJ, Ylä-Herttuala S, et al. Hyperbaric oxygen therapy activates hypoxia-inducible factor 1 (HIF-1), which contributes to improved wound healing in diabetic mice. Wound Repair Regen. 2015 Jan;23(1):98-103.

22. Bosco G, Vezzani G, Enten G, Manelli D, Rao N, Camporesi EM. Femoral condylar necrosis: treatment with hyperbaric oxygen therapy. Arthroplast Today. 2018 Dec;4(4):510-5. 